\title{
Loudness enhancement and summation in pairs or short sound bursts*
}

\author{
J. J. ZWISLOCKI, I. KETKAR, M. W. CANNON, and R. H. NODAR \\ Institu te for Sensory Research, Syracuse University, Syracuse, New York 13210
}

\begin{abstract}
When a pair of short, temporally spaced sound bursts is presented and the listeners are requested to match the loudness of a third, comparison burst to the overall loudness of the pair, a fundamentally different result is obtained than when they are instructed to match the loudness of the comparison burst to that of the second burst in the pair. Loudness-level changes occurring in the first situation are designated as loudness summation; those occurring in the second situation, as loudness enhancement. Some parameters of both phenomena are studied. The results lead to the suggestion of a principle of maximum similarity in stimulus matching and to a reinterpretation of some earlier data.
\end{abstract}

According to Niese (1956) and Schwarze (1963), the loudness of two short tone bursts of the same sound frequency differs little from the loudness of a single burst. A small increment in loudness level, on the order of $3 \mathrm{~dB}$, decays to zero when the bursts stop being temporally fused, that is, within about 50 msec. According to Scharf (1970), the amount of loudness summation depends on the frequency relationship between the bursts in a pair. When their frequencies are within a critical band, the increment in loudness level is on the order of $3 \mathrm{~dB}$; when the frequencies are separated by several critical bands (Zwicker, Flottorp, \& Stevens, 1957), it may reach $10 \mathrm{~dB}$. The increment persists beyond the interval of temporal fusion and seems to decay with a time constant on the order of $200 \mathrm{msec}$. Scharf's results appear consistent with data on loudness of tone complexes (for instance, Zwicker, Flottorp, \& Stevens, 1957), on temporal summation at the threshold of audibility, and on loudness as a function of sound duration (for review and unifying theory, see Zwislocki, 1960, 1969). Nevertheless, Irwin and Zwislocki (1971) obtained data that do not agree entirely with those of Scharf and have aspects in common with the data of Niese and Schwarze.

In order to clarify the situation, we have undertaken several series of experiments with tone bursts. The results of these experiments indicate that the. disagreement among previous investigations is due to various degrees of interaction between two distinct perceptual processes. When a burst pair is followed by a third, comparison burst, the listener may be asked to perform one of the following three loudness-matching tasks: match the loudness of the third burst to the loudness of the first burst, to that of the second burst, or to the total loudness of the burst pair. Depending on experimental conditions, one of the tasks may appear

\footnotetext{
*The material of this article was presented at the 1970 spring meeting (Cannon \& Zwislocki, 1970) and the 1971 fall meeting (Zwislocki \& Ketkar, 1972) of the Acoustical Society of America and is contained in part in Ketkar's 1972 doctoral dissertation. The work was supported in part by ONR Contract N00014-67-A-03780-0003 and in part by NIH Grant NS03950.
}

easier than the others, and some listeners seem to execute it almost independently of instructions.

In our experiments, listeners were asked to match the loudness of the third burst to either the loudness of the second burst or of the burst pair. In order to avoid semantic confusion, we shall call loudness increases referring to the second burst "loudness enhancement," and those referring to the burst pair, "loudness summation."

\section{METHOD}

Monaural triades of $10-\mathrm{msec}$ tone bursts with $5-\mathrm{msec}$ rise and fall times were presented to groups of 4 to 7 listeners. The time interval between the first two bursts was varied, the time interval between the second and third bursts remained at $500 \mathrm{msec}$. The listeners could adjust the loudness level of the third burst by turning a smooth round knob connected to a precision log attenuator. All clues that could indicate to the listener the position of the attenuator setting were carefully avoided, and before each adjustment, the $E$ introduced randomized amounts of attenuation by means of an auxiliary attenuator. When the sound frequencies of the first two bursts were equal, the bursts were presented at the same SPL. When the frequencies were different, the bursts were made individually equal to the loudness level of the third burst. Equality was accepted when the loudness level of the third burst was found to be the same within $2 \mathrm{~dB}$ in the match to either burst. In one experiment in which the first burst consisted of broadband noise, its loudness was made greater than the loudness of the second burst. The loudness level of the third burst obtained in the match to the second burst presented singly was always used as the reference level. The second burst was maintained at $60 \mathrm{~dB} \mathrm{SL}$, except in the experiment with noise, in which it was at approximately $45 \mathrm{~dB}$.

In the main experiment, the sound frequency of the first burst was always at $1 \mathrm{kHz}$, and that of the second, at $4 \mathrm{kHz}$. The sound frequency of the third burst was made equal to either the frequency of the second or of the first burst, or to their geometric mean. In one sequence, the listeners were required to match the loudness of the third burst to that of the second and disregard the first burst; in another, they had to match the loudness of the third burst to the combined loudness of the first two bursts. The E put an unmistakable emphasis on "combined loudness." In one auxiliary experiment, all three bursts had the same sound frequency, in another, the first burst consisted of broadband noise, and the frequency of the second and third bursts was at $1 \mathrm{kHz}$. In both experiments, the listeners were 
required to match the loudness of the third burst to that of the second. 1

\section{RESULTS OF THE MAIN EXPERIMENT}

The results are shown in Fig. 1. Each dot indicates the mean of three adjustments by each of the seven listeners participating in the experiment. The solid lines join the group medians. The data in the three graphs on the left side were obtained with the instruction to match the loudness of the second burst, those on the right side, with the instruction to match the total loudness of the burst pair. Although, in each graph, the parameter values of the burst pair were the same, and only the frequency of the third burst and the instructions were not, the results clearly differ among the conditions.

First, let us consider the two extreme results. The minimum loudness effect appears in the upper left corner, and the maximum, in the lower right corner. The minimum occurred when the listeners attempted to match the loudness of the third burst to the loudness of the second, and the sound frequency of both bursts was the same. Judging from the small variability of the individual data, the task was easy, especially at long time intervals, when the second burst was heard as a separate entity from the first. Very likely, the listeners followed the instructions accurately, and according to our definition, the results refer to loudness enhancement. We must conclude that, except perhaps at very short time intervals, a preceding $1-\mathrm{kHz}$ tone burst does not appreciably enhance the loudness of a following $4-\mathrm{kHz}$ tone burst when both are at the same loudness level. Earlier experiments have shown that approximately th: same result is obtained when the first and the second bursts are at the same sound frequency (Irwin \& Zwislocki, 1971).

The maximum loudness effect occurred when the listeners were instructed to match the loudness of the third burst to the total loudness of the burst pair, and the sound frequency of the third burst was at the geometric mean of the burst-pair frequencies. Apparently, the listeners followed the instructions well, since the obtained $10-\mathrm{dB}$ increment in loudness level is approximately equivalent to doubling of loudness (Stevens, 1955). However, the inter-S variability indicates that the task was more difficult than matching two bursts of equal frequency. Surprisingly, the variability did not decrease at short time intervals at which the bursts in the burst pair appeared to be fused. A similar observation was made by Scharf (1970). Perhaps the most striking outcome of this experiment is the independence of the loudness level of the interburst time interval up to at least $500 \mathrm{msec}$. It leads us to believe that the listeners consciously summated the component loudnesses and adjusted the third burst to match the imaginary loudness of the sum. In any event, the time independence suggests that the process is different from that of temporal summation at the threshold of audibility, which has a time constant on the order of $200 \mathrm{msec}$.

The intermediate results shown on the left side of Fig. 1 under $\mathrm{b}$ and $\mathrm{c}$ may be explained by assuming that, when the frequency of the third burst is different from the frequency of the second, making the loudness match between the two bursts more difficult, listeners tend to respond to the total loudness of the burst pair in the presence of temporal fusion, and to the loudness of the second burst when the fusion breaks down. Such an explanation is consistent with an increase of loudness level on the order of $10 \mathrm{~dB}$ at 0 time interval and no increase at $500 \mathrm{msec}$.

The intermediate results shown on the right side under $d$ and e are more difficult to understand. They indicate that, while the listeners continued to summate the loudness of the burst pair independently of the time interval, the summation became partial when the frequency of the third burst coincided with one of the component frequencies in the burst pair rather than with their geometric mean. This result agrees with observations of Chaves (1971).

To show that the course of the median curves in Fig. 1 reflects typical behavior rather than a statistical accident, we have plotted in Fig. 2 typical results obtained on two listeners. They exhibit two features of interest. First of all, they closely follow the median curves for every experimental condition. In addition, they show more clearly than the median curves pairwise similarities between the left- and right-side graphs of the figure. The similarities indicate that equal frequency relationships between the third burst and the remaining two bursts tend to produce similar results. Independent of instructions, the smallest loudness level resulted when the frequency of the third burst was equal to that of the second burst, and the largest, when it was equal to the geometric mean of the frequencies of the first two bursts. Frequency equality between the third and first bursts produced intermediate results. The main effect of the listener instructions was to change the course of the loudness level as a function of the time interval between the first two bursts. This is especially apparent in the bottom graphs obtained for the frequency of the third burst at the geometric mean of the frequencies of the first two bursts.

\section{RESULTS OF THE FIRST AUXILIARY EXPERIMENT}

The purpose of this experiment was to ascertain that the almost complete absence of loudness enhancement in the main experiment (Figs. 1a and 2a) was not due to the rather substantial frequency difference between the first and second bursts. Consequently, the frequency of the first burst was made equal to the frequency of the second at $4 \mathrm{kHz}$, and the matching of the loudness of the third burst to that of the second repeated on the 
Fig. 1. Change in the loudness level of a third burst, when matched to either the loudness of the second burst in a pair or to the overall loudness of the pair, as a function of the time interval between the bursts in the pair. Loudness level of the third burst, when matched to the loudness of the second burst presented singly, is the reference ordinate. The left side of the figure corresponds to matching the loudness of the second burst, the right side, to matching the overall loudness. The sound frequencies of the bursts are indicated on the graphs. Note that only the frequency of the third burst changes. Dots indicate individual data, lines connect their medians.

Fig. 2. Same as Fig. 1., but for two individual listeners.
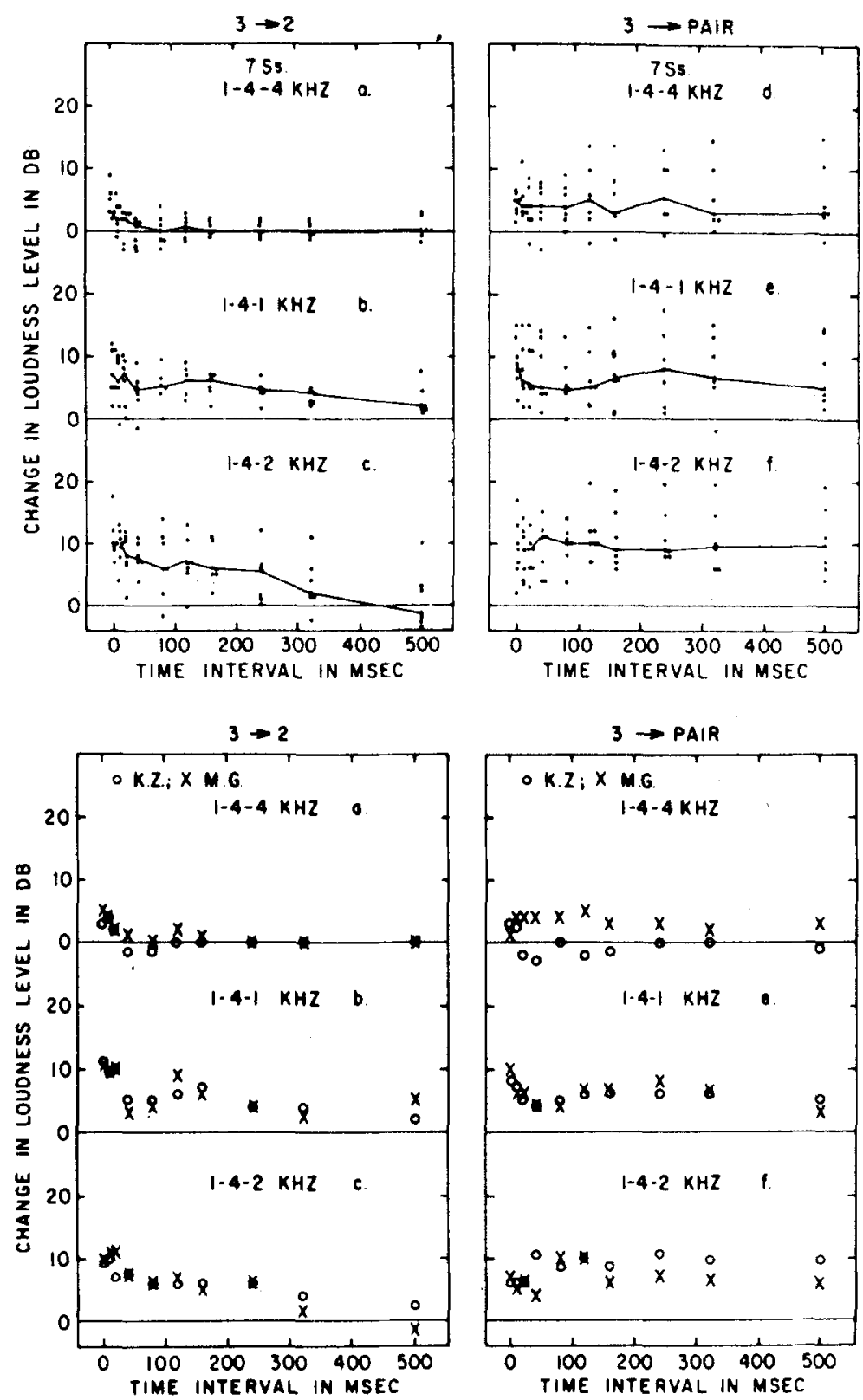

same group of listeners. The median results are shown in Fig. 3 by means of crosses and a dashed line. The circles and solid curves in the same figure have been transferred from Figs. 1a and 1f, for comparison. As can be seen, the frequency change produced only a small effect, whose significance is questionable. Similar results were obtained at several other frequencies (Ketkar, 1972). We conclude, in agreement with earlier experiments (Irwin \& Zwislocki, 1971), that the loudness of a tone burst is not appreciably enhanced by a preceding tone burst of equal loudness, independent of the interburst frequency difference.

\section{RESULTS OF THE SECOND AUXILIARY EXPERIMENT}

The experiment was undertaken to find out if a clear loudness enhancement, as defined in this article, can occur under favorable conditions, and if so, to gain a preliminary insight into its frequency selectivity. To these ends, the first burst was cut out of broadband random noise. The remaining two bursts consisted of $1-\mathrm{kHz}$ sinusoids. The listeners were requested to match the loudness of the third burst to that of the second. In a first series, the loudness of the noise burst was approximately equal to that of the second burst; in a second series, the noise intensity was so adjusted that the SPL in the critical band centered at $1 \mathrm{kHz}$ was equal to the SPL of the second burst.

If the same spectral integration took place in loudness enhancement as had been found in threshold and masking experiments (Feldtkeller \& Zwicker, 1956), the second series should have produced results equivalent to 


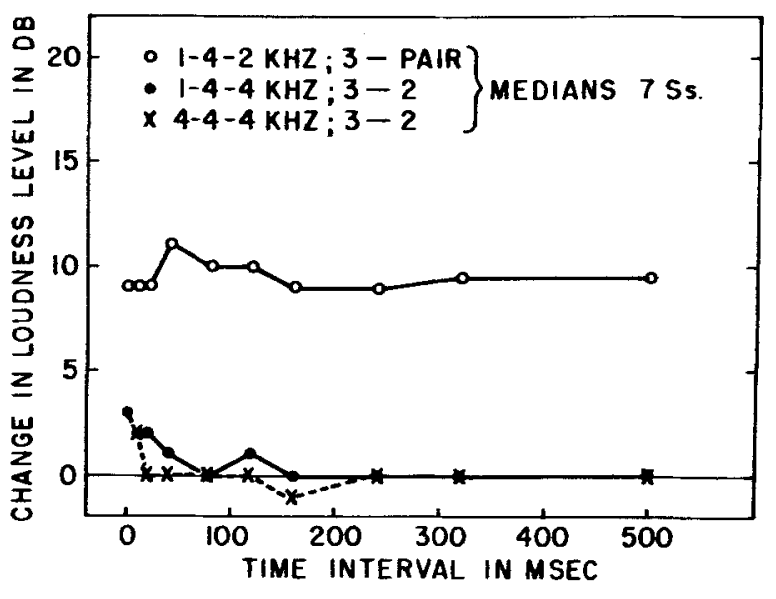

Fig. 3. Change in the loudness level of the third burst when matched to the overall loudness of a burst pair (upper curve), or to the second burst in a pair (lower curves) as a function of the time interval between the first two bursts. The sound frequencies of the bursts are indicated on the graph.

the results shown by the two lower curves of Fig. 3 . The first series should have produced an even smaller effect. Figure 4 shows that this is not what happened. The crosses and the intermittent line indicate the median results obtained with the weaker noise burst whose total loudness was the same as the loudness of the second burst. Comparison with the two lower curves of Fig. 3 suggests that the noise produced a similar effect to that of an equally loud pure tone. The filled circles of the same figure indicate the individual means obtained with the stronger noise. The solid curve connects their medians. It shows that a noise with a critical-band SPL equal to the SPL of the second burst produces a much greater effect than a pure tone burst of equal SPL. In addition, the effect is more complex. At short interburst time intervals, the noise burst reduced the loudness level of the second burst by as much as $10 \mathrm{~dB}$. Since the reduction was found at intervals greater than $20 \mathrm{msec}$, it could not have been produced by a physical interaction between the bursts. At time intervals exceeding $60 \mathrm{msec}$, a long-lasting loudness enhancement took place. It reached a maximum on the order of $4 \mathrm{~dB}$ between 80 and $160 \mathrm{msec}$ and gradually decayed at longer intervals. The slow decay may reflect the 200 -msec constant of temporal integration found in threshold experiments (Zwislocki, 1960). However, a spectral bandwidth greater than one critical band seems to have intervened. Whether the total loudness of the noise was involved or a fraction of it, cannot be decided on the basis of our data. Additional experiments are required for the determination of the effective bandwidth in loudness enhancement.

\section{CONCLUSIONS}

According to our results, at least two processes must be considered in dealing with loudness effects in pairs of short tone bursts. One concerns the loudness of each individual burst, and has been designated as loudness enhancement; the other concerns the total loudness of the burst pair, and has been designated as loudness summation. Whether one or the other is measured depends on the instructions to the listeners and on the sound frequency of the reference burst relative to the component frequencies of the burst pair. Listeners tend to match the reference burst to that perceptual aspect of the burst pair which most resembles the perception of the reference burst. We may designate this tendency as the principle of maximum similarity. Unless the task assigned to the $S$ is consistent with the principle, ambiguous results are likely to result.

We suspect that some past experiments on temporal loudness effects did not observe the principle of maximum similarity and have led to the existing inconsistencies. Two examples may elucidate the situation. They are illustrated in Fig. 5. As a reference, the solid curve reproduces our data of Fig. $1 \mathrm{c}$, and the intermittent one, those of Fig. 1f, both obtained with the frequency of the reference burst at the geometric mean of the burst-pair frequencies. The solid line resulted when the listeners were instructed to match the loudness of the second burst, the intermittent line, when they were explicitly instructed to match the loudness of the burst pair. We have pointed out that the data of the solid curve probably resulted from a gradual shift of listeners' criterion. They probably matched the loudness of the burst pair at short time intervals, when the bursts appeared fused, and gradually changed to matching the loudness of the second burst, when the fusion began to break down. The circles in Fig. 5 reproduce Scharf's (1970) data obtained under analogous stimulus

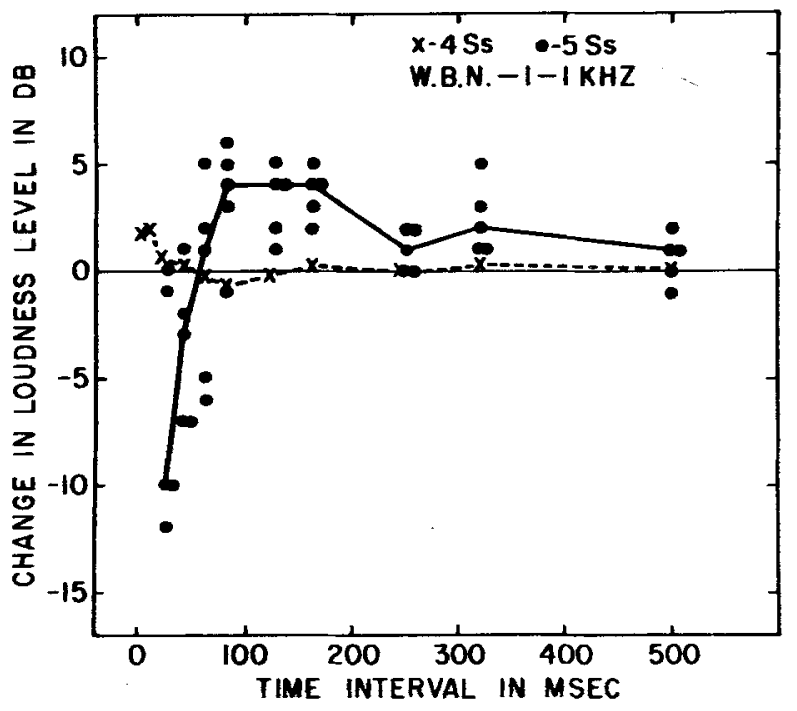

Fig. 4. Loudness enhancement of a tone burst produced by a preceding noise burst, as a function of time interval between the bursts. Crosses correspond to median data obtained with a weaker noise, circles, to individual data obtained with a stronger noise: The solid line connects the medians of the individual data. 
Fig. 5. Loudness-level change of the third burst matched in loudness to a burst pair or the second burst of the pair (solid curve) under different experimental conditions. Curves from Fig. 1, circles from Scharf (1970), crosses from Irwin and Zwislocki (1971).

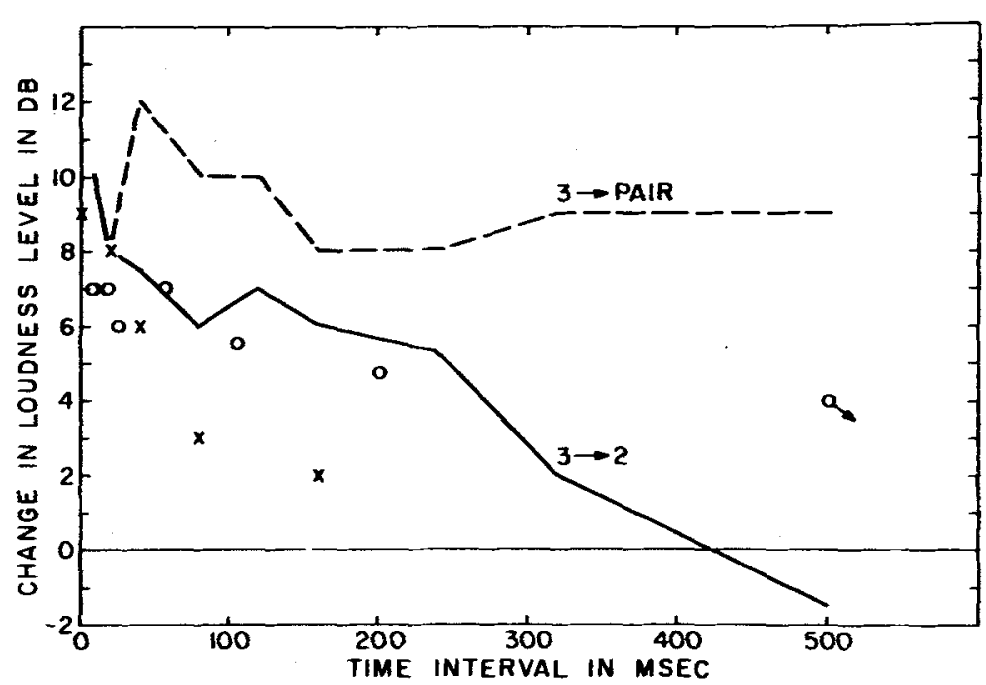

conditions. His instruction to the listeners was to match the loudness of the burst pair. However, he seems not to have emphasized the matching criterion to the point where his listeners would maintain it at long time intervals, and his data follow the general trend of the solid curve rather than that of the intermittent one. An even greater departure from the intended criterion is suggested by the crosses in Fig. 5, which reproduce corresponding results of Irwin and Zwislocki (1971). In their experiments, the instructions to the listeners were probably similar to those given by Scharf, but the task of matching the loudness of the burst pair was rendered more difficult by making the frequency of the reference burst equal to the frequency of the second burst.

In more general terms, all the results included in Fig. 5 converge at short time intervals, suggesting that the listeners tended to match the overall loudness of the burst pair when the bursts appeared fused. At long time intervals, the listeners seem to have changed their behavior to matching the loudness of the second burst, unless matching the overall loudness of the burst pair was strongly emphasized to them. In different terms, it appears easy to determine temporal loudness summation of burst pairs at short interburst time intervals, but very difficult at long intervals.

Concerning loudness enhancement, we have to conclude on the basis of Figs. 1, 2, 3, and 4, that it is almost completely absent, independent of frequency relationships between the two bursts in a pair, as long as both bursts have the same loudness. This conclusion is consistent with the results of Irwin and Zwislocki (1971). When the loudness of the first burst exceeds that of the second, a clear loudness enhancement occurs (Fig. 4). This conclusion finds a strong confirmation in recently published papers of Galambos and his coworkers $(1971,1972)$.

\section{REFERENCES}

Cannon, M. W., \& Zwislocki, J. J. Loudness summation of pairs of short sounds. Journal of the Acoustical Society of America, $1970,48,71(\mathrm{~A})$.
Chaves, J. F. Two-tone loudness summation and the spectra of comparison sounds. Journal of the Acosutical Society of America, 1971, 50, 144(A).

Feldtkeller, R, \& Zwicker, E. Das Ohr als Nachrichtenempfä́nger. Stu ttgart: S. Hirzel, 1956.

Galambos, R., Bauer, J., Picton, T., Squires, K., \& Squires, N. Loudness enhancement following contralateral stimulation. Journal of the Acoustical Society of America, 1972, 52, 141(A).

Galambos, R., Bauer, I., Picton, T., Squires, K., \& Squires, N. Loudness enhancement following contralateral stimulation. Journal of the Acoustical Society of America, 1972. 52. 1127-1130.

Irwin, R. J., \& Zwislocki, J. J. Loudness effects in pairs of tone bursts. Perception \& Psychophysics, 1971, 10, 189-192.

Ketkar. I. The effects of frequency and temporal separation on the loudness of pairs of short tone bursts. Doctoral dissertation, Syracuse University, 1972.

Niese, $H$. Vorschlag fuer die Definition und Messung der Deutlichkeit nach subjektiven Grandlagen. Hochfrequenztechnik und Electroakustik, 1956, 65, 4-15.

Scharf, B. Loudness and frequency selectivity at short durations. In R. Plomp and G. F. Smoorenburg (Eds.), Frequency analysis and periodicity detection in hearing. Leiden: Sijthoff, 1970.

Schwarze, D. Die Loutstaerke von Gausstoenen. Doctoral dissertation, Technische Universitaet, Berlin, 1963 (cited by Scharf, 1970).

Stevens, S. S. The measurement of loudness. Journal of the Acoustical Society of America, 1955, 27, 815-829.

Zwicker, E. Flottorp, G., \& Stevens, S. S. Critical bandwidth in loudness summation. Journal of the Acoustical Society of America, 1957, 29, 548-557.

Zwislocki, J. J. Theory of temporal auditory summation. Journal of the Acoustical Society of America, 1960, 32, 1046-1060.

Zwislocki, J. J. Temporal summation of loudness: An analy sis. Journal of the Acoustical Society of America, 1969, 46, 431-4.41.

Zwislocki, J. J., \& Ketkar, I. Loudness enhancement and summation in pairs of short sound bursts. Journal of the Acoustical Society of America, 1972, 51, 140(A).

\section{NOTE}

1. In order to estimate the intralistener variability, listeners made 10 loudness matches at selected stimulus conditions in pilot experiments. We found that most interquartile ranges of the matches remained under $5 \mathrm{~dB}$ and decreased to about $2 \mathrm{~dB}$ for the easier conditions. Because of the small variability, the number of matches was decreased to three in the main experiment and the first auxiliary experiment. This made it possible to use a large number of parameter values without making the results of interest ambiguous. In the second auxiliary experiment, with a smaller number of listeners (four and five), each listener made eight loudness matches per stimulus each listener made eight loudness matches per stimulus
condition. The individual data of Figs. 1,2 , and 4 are represented by the means of the loudness matches. Spot checks have indicated that their standard errors are smaller than comparable measures of interlistener variability.

(Received for publication January 31, 1973; revision received March $1,1974$. 